\title{
Improved localization algorithm with FDOA measurements
}

\author{
Ya-Xiao Niu, Shan-Wei Shi, Chun-Dong $\mathrm{Qi}^{\dagger}$ and Zhi-Jie Zheng \\ School of information and electronics, Beijing Institute of Technology \\ Beijing, China \\ E-mail:dong_bit@bit.edu.cn
}

\begin{abstract}
Localization with FDOA measurements is an important passive location method. Traditional solution to nonlinear equations is Newton's iterative method. However, this method may encounter convergence problems. This paper proposes an algorithm that can find initial estimates by dividing target area into girds and improve Newton's iterative method by combining a grid search. A simulation is given to verify the algorithm.
\end{abstract}

Keywords: passive location; FDOA; Newton’s iterative.

\section{Introduction}

If independent sensors in space can receive signals emitted by a single source and measure Doppler-shifted frequencies, it is possible to locate the source based on the differences of Doppler-shifted frequencies between sensors. Known as Frequency Difference of Arrival (FDOA), it is a typical method for passive localization, and becomes an increasingly important technical solution to electronic reconnaissance[1]. But it is difficult to solve the nonlinear equations of FDOA by algebraic methods [2]. As it requires solving nonlinear equation, where analysis method does not work, Newton's iterative method is an important approach available. However, this approach requires initial estimate [3]. Nonlinear equations usually have more than one solution and different initial estimates will converge to different solutions. Consequently, poor selection of initial estimates may miss some solutions [4].

A sufficient condition of initial estimates selection is proposed in Reference [3]. That is, suppose $f(x)=0$, if $x 0$ is selected as an initial estimate and $f\left(x_{0}\right) \square f^{\prime \prime}\left(x_{0}\right)>0$, Newton's method will converge.However, this approach may still miss some solutions, which restricts its application.

This paper develops analgorithmfor satellite platform to solve this problem and better locate ground targets. 


\section{Principle of FDOA}

In a scenario where threesatellites are used to positionan unknown emitter source,located at $\mathbf{R}_{\mathrm{T}}$, with FDOA measurements, let $\mathbf{R}_{\mathrm{i}}$ and $\mathbf{V}_{\mathrm{i}}$ be actual position and velocity of the $i^{\text {th }}$ satellite, $i=0,1,2$, then the frequency of signal fi received by the $i^{\text {th }}$ satellite is [5]:

$$
f_{\mathrm{i}}=f_{c}+\frac{f_{c}}{c} \mathbf{V}_{i} \cdot \frac{\mathbf{R}_{i}-\mathbf{R}_{\mathrm{T}}}{\left|\mathbf{R}_{i}-\mathbf{R}_{\mathrm{T}}\right|}
$$

Where $f_{c}$ is carrier frequency and $c$ is speed of light. By making the difference of $\mathrm{f}_{\mathrm{i}}$ we get the differences of Doppler-shifted frequency as follows:

$$
\left\{\begin{array}{l}
f_{1}-f_{0}=\frac{f_{c}}{c}\left(\frac{\mathbf{V}_{0} \square\left(\mathbf{R}_{0}-\mathbf{R}_{T}\right)}{\left.\mid \mathbf{R}_{0}-\mathbf{R}_{T}\right) \mid}-\frac{\mathbf{V}_{1} \sqsubset\left(\mathbf{R}_{1}-\mathbf{R}_{T}\right)}{\left.\mid \mathbf{R}_{1}-\mathbf{R}_{T}\right) \mid}\right) \\
f_{2}-f_{0}=\frac{f_{c}}{c}\left(\frac{\mathbf{V}_{0} \square\left(\mathbf{R}_{0}-\mathbf{R}_{T}\right)}{\left.\mid \mathbf{R}_{0}-\mathbf{R}_{T}\right) \mid}-\frac{\mathbf{V}_{2} \square\left(\mathbf{R}_{2}-\mathbf{R}_{T}\right)}{\left.\mid \mathbf{R}_{2}-\mathbf{R}_{T}\right) \mid}\right) \\
x^{2}+y^{2}+\frac{z^{2}}{1-e^{2}}=a^{2}
\end{array}\right.
$$

Where a is equatorial radius and e is ellipticity of Earth.

\section{Confirmation of Initial Estimates}

As the $3^{\text {rd }}$ equation is Earth model, the roots of the (3) must be on Earth surface if they exist. The $1^{\text {st }}$ and $2^{\text {nd }}$ equations intersect Earth surface at closed equal frequency difference contours [1].

According to the $1^{\text {st }}$ equation, the equal frequency difference contours can be based on each point on the ground relative to Satellite 0 and Satellite 1. Similarly, another figure of equal frequency difference contours can be obtained based on the 2nd equation. The intersection points of the two contours are the solutions of the equations. As shown in Figure 2, two intersection points of the contours, $\mathrm{f} 1$ and $\mathrm{f} 2$, indicate that there are 2 solutions in the given scenario.

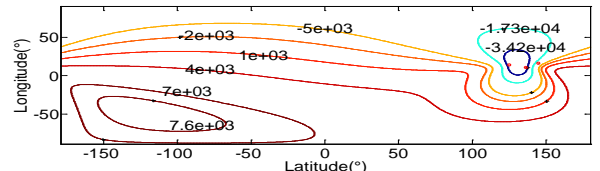

Fig. 1. Equal frequency difference contours (ECEF coordinates need to be transferred to geodetic coordinates)

But actually, there is only one reasonable solution. The others are extraneous and need to be eliminated by other means. When Newton's method is used, it is possible that the initial estimates selected just converge to the extraneous solutions which will be eliminated later. In that case, the source 
cannot be located because the real solution is missed. To avoid this, it is important to find all possible solutions when equations are solved.

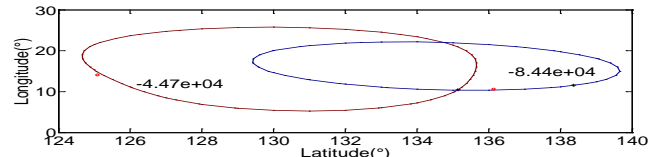

Fig. 2. Two real frequency difference contours

\subsection{When the initial estimates converge to the solution}

The target area is to be divided into grids to analyze the distribution of the solutions. If the grids are small enough, real frequency difference contours will go through the grid where the target is in. The value of the grid points aroundthe contour is greater or smaller than the real frequency difference. One contour can divide four points of a grid into two parts- each part with two pointsor one part with one point and the other with three. When two contours divide one grid, there are three scenarios, as shown in Fig. 3.
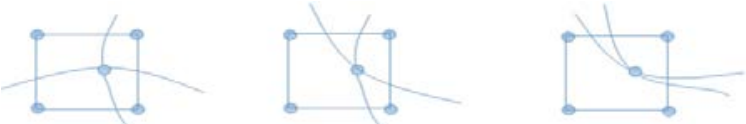

Fig. 3. Three scenarios when two contours divide one grid

On this basis,suppose there is a point with the coordinate of $\mathrm{P}(\mathrm{i}, \mathrm{j})$, its frequency difference between Satellite 0 and Satellite 1 is $f_{1}(i, j)$ and that between Satellite 0 and Satellite 2 is $f_{2}(i, j)$, where $i$ and $j$ correspond to longitude and latitude respectively.

Suppose,

$$
\begin{aligned}
& A_{1}=\left\{f_{1}(i, j), f_{1}(i+\Delta i, j), f_{1}(i, j+\Delta j), f_{1}(i+\Delta i, j+\Delta j)\right\} \\
& A_{2}=\left\{f_{2}(i, j), f_{2}(i+\Delta i, j), f_{2}(i, j+\Delta j), f_{2}(i+\Delta i, j+\Delta j)\right\}
\end{aligned}
$$

Where $\Delta \mathrm{i}$ and $\Delta \mathrm{j}$ are longitude and latitude's interval when dividing target area into grids.

If the number of elements in Set $A_{1}$ greater than $\Delta f_{1}$ is in [1,3] and the number of elements in Set $A_{2}$ greater than $\Delta f_{2}$ is in [1,3], it is highly possible that the target is located in the grid, and he center of the gird can be taken as an initial estimate.

$$
\left\{\begin{array}{l}
\left|\left\{x: x \in A_{1} \wedge x>\Delta f_{1}\right\}\right| \in[1,3] \\
\left|\left\{y: y \in A_{2} \wedge y>\Delta f_{2}\right\}\right| \in[1,3]
\end{array}\right.
$$


However, the target area cannot be divided into infinitely small grids due to the load of computation.So in a real case, only judging by (5) may lead to missing or extraneous initial estimate.

\subsection{Missing initial estimates}

In Fig. 4a and Fig. 4b, a contour is totally or partly in a grid but does not enclose any grid points. In Fig. 4c, two contours intersect at two points in one grid. All of the three situationsare because the grid too big. If it is small enough, the scenarios in Fig. 3 can be obtained.
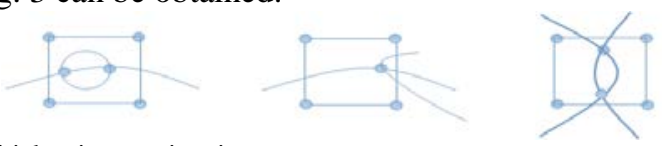

Fig. 4 . Missing initial estimates situation

Under the circumstances of Fig. 4a, if the directions of frequency difference at four grid points tend to point inside or outside of a grid, it is likely that a closed contour is completely in that grid. Then the following equations can be used to measure the trends,

$$
\left\{\begin{array}{l}
{\left[f_{i}(i-\Delta i, j-\Delta j)-f_{i}(i, j)\right] *\left[f_{i}(i+2 \Delta i, j+2 \Delta j)-f_{i}(i+\Delta i, j+\Delta j)\right]>0} \\
{\left[f_{i}(i-\Delta i, j+2 \Delta j)-f_{i}(i, j+\Delta j)\right] *\left[f_{i}(i+2 \Delta i, j-\Delta j)-f_{i}(i+\Delta i, j)\right]>0}
\end{array}\right.
$$

where $\mathrm{i}=1$ or 2 .

If one frequency difference contour satisfies(6)and another satisfies(6) or (5), the center of the grid is a possible initial estimate.

As for the case in Fig. 4b, its neighboring grids will be regarded as possible initial estimates. By Newton iterative method, the initial estimates will converge to real targets because initial estimates and targets are very close.

In the scenario in Fig. 4c, two solutions exist in one grid but only one initial estimate is chosen (5). By Newton's iterative method, the other solution is missed. To solve this problem, the grid, where the original initial estimate is located, is bisected on two dimensions. If another target is found in a new round of Newton's iteration, that is the solution missed before. Otherwise, there is only one target in the grid.

\subsection{Extraneous initial estimates}

In some cases(Fig. 5), the target is not in a grid but the equation set (5) is satisfied which leads to extraneous solution. The reason for that is that the grid is too small.In the case of Fig. 5a, a real target is nearby and the extraneous initial estimate will converge to it by Newton's iterative method; while in the 
case of Fig. 5b, as the equations have no solution, the extraneous initial estimate cannot converge.

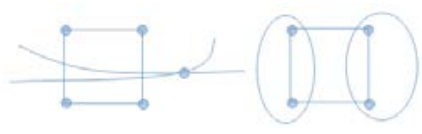

Fig. 5. Extraneous initial estimates situation

\section{Simulations}

Suppose an emitting source has carrier frequency of $3 \mathrm{GHz}$ and coordinate of $\left(135.12^{\circ} \mathrm{E}, 10.33^{\circ} \mathrm{N}\right)$. There are three satellites whose orbit is $1000 \mathrm{~km}$ high . Data, produced by Satellite Tool Kit at certain timing when the satellite bottom points are $\left(125.08^{\circ} \mathrm{E}, 14.08^{\circ} \mathrm{N}\right),\left(136.14^{\circ} \mathrm{E}, 10.63^{\circ} \mathrm{N}\right)$ and $\left(144.62^{\circ} \mathrm{E}, 16.37^{\circ} \mathrm{N}\right)$, are selected.

If a point is chosen as initial estimate randomly for traditional Newton's iterative method, the convergence distribution is shown in Fig. 7.

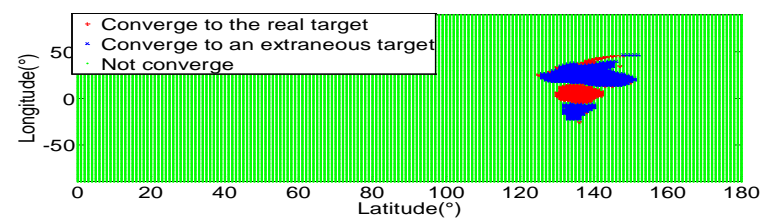

Fig. 7 Initial estimate randomly for Newton's iterative method

The green area is non-convergent and the red and blue areas are convergent. The initial estimates in red area converge to the real emitting source, while those in blue area converge to anextraneous solution.

Then the proposed algorithm is used.Firstly, the target area is divided into $360 * 180$ gridswith interval of 1 degree. Then two frequency difference contours are obtained and the possible initial estimates are listed in Tab. 1.

Table 1:Initial estimates.

\begin{tabular}{ccc}
\hline No & Longitude $\left({ }^{\circ}\right)$ & Latitude $\left({ }^{\circ}\right)$ \\
\hline 1 & 129.500 & 14.500 \\
2 & 129.500 & 15.500 \\
3 & 130.500 & 12.500 \\
4 & 130.500 & 13.500 \\
5 & 130.500 & 14.500 \\
6 & 134.500 & 21.500 \\
7 & 134.500 & 22.500 \\
8 & 135.500 & 10.500 \\
9 & 135.500 & 15.500 \\
10 & 135.500 & 16.500 \\
11 & 135.500 & 21.500 \\
\hline
\end{tabular}


Two sets of solutions, $\left(134.767^{\circ} \mathrm{E}, 22.028 .33^{\circ} \mathrm{N}\right)$ and $\left(135.120^{\circ} \mathrm{E}\right.$, $10.330^{\circ} \mathrm{N}$ )are obtained by applying Newton's method on the above estimates.Therefore, the proposed algorithm overcomes the disadvantages of Newton's method andfinds all the possible solutions, but does not increase too much computational complexity.

\section{Conclusions}

Iterative method is the only way to solve complex nonlinear equations for locating targets with FDOA measurements. In this paper, an algorithm is developed to find valid initial estimates so that we can avoid convergence problem and missing solutions. Furthermore, this algorithm can also be applied in other location scenarios which contain Earth model equation, such as location with TDOA measurements based on three satellites and location with combined TDOA/FDOA measurements.

\section{References}

1 Xu,H., LV S., Han, T.:'Doppler Frequency Difference Based ThreeSatellite Passive Algorithm and Its Precision Analysis', Journal of Astronautics, 2010.31,(7), pp. 1832-1837,doi:10.3873/j.issn.10001328.2010.07.021

2 Ho,K. C. and Chan,Y. T.:'Geolocation of a known altitude object from TDOA and FDOA measurements', IEEE Trans. Aerosp. Electron. Syst., 1997,,33, (7), pp. 770-783, doi:10.1109/7.599239

3 Chan,Y.T.: 'A 1-D search solution for localization from frequency measurements', IEEE Journal of Oceanic Engineering, 1994,19, ( 3), pp. 431-437,, doi:10.1109/48.312919

4 Liu, H.:'Newton Iteration Method for Nonlinear Equation Solutions and Its Application', Journal of Chongqing Institute of Technology (Natural Science Edition), 2007, 21,(8), pp 95-98

5 Lu, X., Zhu, W. and Zheng,T.: 'Passive location using Doppler FDOA method', Aerospace Electronic Warfare, 2008, 24, (3),pp. 40-43

6 Xia, N., Zhang, S. Mu, H.:'Space TT\&C System',(National Defense Industry Press, Beijing,China,2002)

7 Wei, H., Peng, R., Wan Q.,etal:'Multidimensional scaling analysis for passive moving target localization with TDOA and FDOA measurements', IEEE Trans. Signal Process., 2010,,58,(3), pp. 16771688,doi:10.1109/TSP.2009.2037666 
8 Yang, J. Liu, C.: 'Iteration FDOA Location algorithm and its performance analysis', Journal of Xidian University, 2013, 40,(5), pp. 814,doi:10.3969/j.issn.1001-2400.2013.05.002 